\title{
HTLV-1 Tax Specific CD8+ T cells express low levels of Tim-3 in HTLV-1 infection: implications for progression to neurological complications
}

\author{
Fabio E Leal ${ }^{1,2^{*}}$, Lishomwa C Ndhlovu ${ }^{3}$, Aaron M Hasenkrug ${ }^{3}$, Aashish R Jha ${ }^{3}$, Karina I Carvalho ${ }^{1}$, \\ ljeoma G Eccles-James ${ }^{3}$, Fernanda R Bruno ${ }^{1}$, Raphaella G Vieira ${ }^{3}$, Vanessa A York ${ }^{3}$, R Brad Jones ${ }^{4}$, Yuetsu Tanaka ${ }^{5}$, \\ Walter K Neto ${ }^{6}$, Sabri S Sanabani ${ }^{6}$, Mario A Ostrowski ${ }^{4}$, Aluisio C Segurado ${ }^{2}$, Douglas F Nixon ${ }^{3}$, Esper G Kallas ${ }^{1,2}$
}

From 15th International Conference on Human Retroviruses: HTLV and Related Viruses Leuven and Gembloux, Belgium. 5-8 June 2011

\section{Background}

Most patients with HTLV-1 infection are asymptomatic, however $3 \%$ of individuals develop a progressive neurological disorder, HTLV-1 associated myelopathy/tropical spastic paraparesis (HAM/TSP). Factors leading to this complication are not well defined, but are associated with high levels of pro-virus and cytolytic T cells. During chronic viral infections, virus-specific CD8+ T cells undergo altered patterns of differentiation and can become restrained from effector activity. We hypothesized that suppression of immune receptors $\mathrm{T}$ cell immunoglobulin and mucin domain-containing protein 3 (Tim-3) and PD-1 would result in partial reversal of a $\mathrm{T}$ cell restraint profile. In turn this increased cytolytic activity may mediate neuro-immunopathology of HAM/ TSP.

\section{Methods}

We investigated the expression of Tim-3 and PD- 1 on T cells in 22 serially recruited HTLV-1 asymptomatic and HAM/TSP patients and 7 HTLV-1-seronegative matched controls, their distribution on HTLV-1-specific $\mathrm{T}$ cells and the relationship with $\mathrm{T}$ cell function.

\section{Results}

Using flow cytometry, we found that patients with HAM/TSP had significantly lower levels of Tim-3+ PD1- expressing CD8+ $(\mathrm{p}=0.002)$ and $\mathrm{CD} 4+(\mathrm{p}=0.004) \mathrm{T}$ cells compared to healthy uninfected controls. HTLV-1
Tax11-19, HLA-A*02 restricted CD8+ T cells among HAM/TSP individuals expressed markedly lower levels of Tim-3. Furthermore, we found that the frequency of Tim-3 expressing Tax11-19 specific CD8+ T cells inversely correlated with the number of IFN- $\gamma$ secreting cells in response to the Tax Tax11-19 peptide.

\section{Conclusions}

We propose that this low expression of Tim-3 on HTLV-1 Tax-specific T cells may lead to persistent and deleterious effector $\mathrm{T}$ cell pool, resulting in more inflammation and disease progression.

\section{Author details \\ 'Division of Clinical Immunology and Allergy School of Medicine, Universidade de Sao Paulo, Brazil. ${ }^{2}$ Department of Infectious Diseases School of Medicine Universidade de Sao Paulo, Sao Paulo, Brazil. ${ }^{3}$ Division of Experimental Medicine, Department of Medicine, University of California, San Francisco, CA, 94110 , USA. ${ }^{4}$ Department of Immunology , University of Toronto, Medical Sciences Building, King s College Circle, Toronto, ON M5S 1A8, Canada. ${ }^{5}$ Department of Immunology, University of the Ryukyus, Okinawa, 903-0215, Japan. ${ }^{6}$ Molecular Biology Laboratory, Fundação Pro- Sangue, Hemocentro de Sao Paulo, Sao Paulo, Brazil.}

Published: 6 June 2011

doi:10.1186/1742-4690-8-S1-A94

Cite this article as: Leal et al: HTLV-1 Tax Specific CD8+ T cells express low levels of Tim-3 in HTLV-1 infection: implications for progression to neurological complications. Retrovirology 2011 8(Suppl 1):A94.

\footnotetext{
* Correspondence: fabioit@meridionalrd.com

'Division of Clinical Immunology and Allergy School of Medicine,

Universidade de Sao Paulo, Brazil

Full list of author information is available at the end of the article
}

(c) 2011 Leal et al; licensee BioMed Central Ltd. This is an open access article distributed under the terms of the Creative Commons 